\title{
Peptidomimetics have potential
}

A series of peptides that specifically interact with the DNA binding domain of ERG (ETS) have been identified. These ERG inhibitory peptides (EIPs) and their derived peptidomimetics bound ERG with high affinity, resulting in ERG protein degradation and, therefore, offer a promising therapeutic option for treating tumours that express the TMPRSS2-ERG fusion protein.

Wang and colleagues identified 12 unique peptides that interact with ERG using iterative screening of a phage display random peptide library. EIP1 and EIP2 bound strongly to the ERG ETS domain and the investigators then identified the specific interaction residues as a nine-amino-acid stretch of the ETS domain. Synthetic peptides of EIP1 and EIP2 also had high binding affinities for ERG, which was disrupted by a point mutation Tyr373Ala in ERG.
EIPs disrupted ERG-AR and ERG-DNA-PKc interactions in a dose-dependent manner and bound to TMPRSS2-ERG. EIP1 conjugated with HIV-TAT to facilitate cell uptake colocalized with ERG in the nucleus and the cytoplasm and inhibited VCaP and ERG-overexpressing RWPE-1 cell invasion. However, this peptide had no effect on DU145 or PC3 cells, which are negative for ERG fusion products.

Retroinverso versions of EIP1 and EIP2 had similar ERG inhibitory properties to their parental peptides and also inhibited ERG-driven cell proliferation and invasion in vitro. These peptides also disrupted ERGAR and ERG-DNA-PKc interactions and destabilized ERG $24 \mathrm{~h}$ after treatment. ERG and DNA-PKc enrichment to ERG-regulated genomic loci was inhibited by the retroinverso peptides and resulted in dysregulation of
ERG-regulated genes, similar to the effects of ERG knockdown.

Retroinverso EIP1 inhibited ERGmediated invasion and intravasation of TMPRSS2-ERG-positive $\mathrm{VCaP}$ cells in chicken embryos.

In vivo, treatment with a single dose of retroinverso EIP1 blocked the interaction of ERG-PKcs and reduced tumour growth in $\mathrm{VCaP}$ xenografts in mice. Mice receiving $25 \mathrm{mg} / \mathrm{kg}$ of retroinverso EIP1 had no signs of tumour recurrence 60 days after treatment. This effect was specific to ERG-fusion-positive xenografts.

These data show that peptidomimetics of EIPs have considerable therapeutic potential for treating ERG-fusion-positive prostate cancer.

Louise Stone

ORIGINAL ARTICLE Wang, X. et al. Development of peptidomimetic inhibitors of the ERG gene fusion product in prostate cancer. Cancer Cell http://dx.doi.org/10.1016/j.ccell.2017.02.017 (2017)

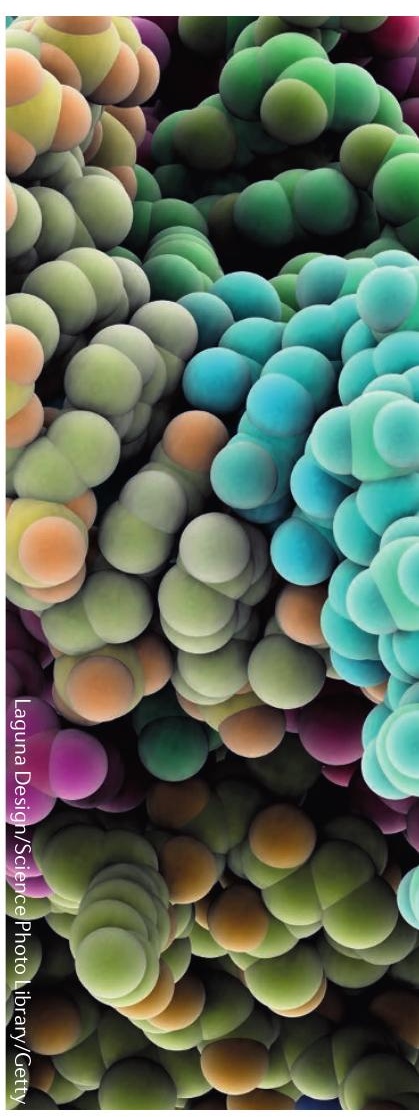

